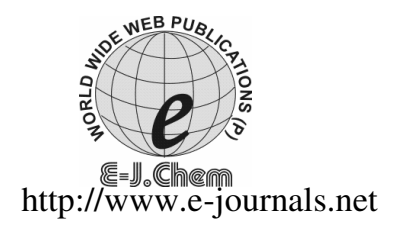

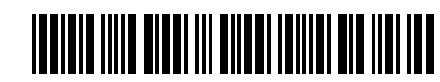

ISSN: 0973-4945; CODEN ECJHAO

E-Journal of Chemistry 2011, 8(2), 665-670

\title{
Microwave Induced Improved Synthesis of Some Novel Substituted 1, 3-Diarylpropenones and their Antimicrobial Activity
}

\author{
K. L. AMETA*, BIRESH KUMAR and NITU S. RATHORE \\ Department of Chemistry, FASC \\ Mody Institute of Technology \& Science \\ (Deemed University) Lakshmangarh - 332311, Rajasthan, India \\ klameta77@yahoo.co.in
}

Received 31 August 2010; Accepted 24 November 2010

\begin{abstract}
Application of solid support, solvent free reaction condition and a dynamic microwave power system in the chemical synthesis of some novel 1, 3-diaryl-propenones has been described. A series of chalcones (3a-h) were synthesized by the condensation of 4-hydroxy-3,5-dinitroacetophenone with various substituted aromatic aldehydes in presence of montmorrilonite $\mathrm{K} 10$ as a catalyst and solid support media under microwave irradiation. The protocol offers several advantages such as simple procedure, fast reaction rate, mild reaction condition, eco-friendly and improved yield as compared to conventional methods. These compounds have been screened for their antibacterial and antifungal activities against different microorganisms. The structures of novel synthesized compounds have been established on the basis of elemental analysis, ${ }^{1} \mathrm{H}$ NMR, ${ }^{13} \mathrm{C}$ NMR and IR spectral data.
\end{abstract}

Keywords: Microwave irradiation, 1,3-Diarylpropenones, Acetophenone, Aromatic aldehydes, Montmorrilonite K10.

\section{Introduction}

Due to the rapid development of bacterial resistant to anti bacterial agents, it is vital to discover novel scaffold for the design and synthesis of the new antibacterial agents to help in the battle against pathogenic microorganism. 1,3-Diarylpropenones (chalcones) represent an essential group of natural as well as synthetic products and some of them possess wide range of pharmacological activities such as antitumor ${ }^{1}$, antibacterial ${ }^{2}$, anticancer ${ }^{3}$, antitubercular ${ }^{4}$, anti-inflammatory ${ }^{5}$, antioxidant ${ }^{6}$, antimalarial ${ }^{7}$ and antileshmanial ${ }^{8}$, etc. The presence of reactive $\alpha, \beta$-unsaturated keto group in the 1,3 diarylpropeneones is found to be responsible for their biological activity. In addition, these compounds are of a great interest due to their use as starting material in the synthesis of a series of a heterocyclic ${ }^{9}$, carbocyclic $^{10}$ and flavonoids ${ }^{11}$. Moreover these are important intermediates in many addition reactions of nucleophiles due to inductive polarization of carbonyl group at the $\beta$-position. Several strategies for the synthesis of the system based on the formation of carbon-carbon bond have been reported. Among them the direct aldol condensation and Claisen Schmidt condensation still occurs prominent 
position. The main method for the synthesis of chalcones is the classical Claisen-Schmidt condensation in the presence of aqueous alkali ${ }^{12}, \mathrm{Ba}(\mathrm{OH})_{2},{ }^{13}$ microwave irradiation ${ }^{14-17}$, ultra sound irradiation ${ }^{18}$. However many of this methods suffered from harsh reaction conditions, toxic reagents, strong acidic / basic conditions, prolonged reaction time, poor yield and low selectivity. Although, several modification have been made to counter these problems. There is still a need for the development of selective and better strategies for the synthesis of $\alpha, \beta$-unsaturated carbonyl compounds. Keeping in view of these finding and in continuation of our interest in the chemistry of chalcones $^{19-24}$ and usefulness of microwave in various organic synthesis ${ }^{25-27}$, herein we describe a simple and convenient method for the synthesis of chalcones, using Montmorrilonite K10 clay under microwave irradiation in solvent free environment, with improved yields and short reaction time.

\section{Experimental}

All melting points (m.ps.) were determined in open capillaries on Veego (VMP - PM) melting point apparatus and are uncorrected. IFB 20S2 Microwave oven with ten power levels was employed for the synthesis of these compounds. The purity of the compounds was routinely checked by thin layer chromatography (TLC) with Silica Gel G (Merk). The instruments used for spectroscopic data are; IR-FTTR spectrophotometer Perkin Elmer RX $1(\mathrm{KBr}),{ }^{1} \mathrm{HNMR} \&{ }^{13} \mathrm{C}$ NMR $\left(\mathrm{CDCl}_{3}\right.$ - solvent) on $500 \mathrm{MHz}$ FT-NMR spectrometer Bruker AV III and elemental analysis was carried out on a Carlo Erba 1108 analyzer and were within the $\pm 0.5 \%$ of the theoretical values. Column chromatography was performed on silica gel (Merck, 60-120 mesh).

\section{General procedure for the preparation of chalcones $(\mathbf{3 a}-\boldsymbol{h})$}

To a solution of 4-hydroxy-3,5-dinitroacetophenone $(0.01 \mathrm{~mol})$ and substituted aromatic aldehyde $(0.01 \mathrm{~mol})$ in ethanol $(5 \mathrm{~mL})$ taken in $100 \mathrm{~mL}$ borosil flask, was added montmorrilonite $\mathrm{K} 10$ clay (4g). The mixture was uniformly mixed with glass rod and air dried to remove the solvent. Adsorbed material was irradiated inside a microwave oven for 4-6 min. at medium power level $(600 \mathrm{~W})$. After the completion of the reaction (monitored by TLC), the reaction mixture was cooled at room temperature and the product was extracted with ethanol $(2 \times 20 \mathrm{~mL})$. Removal of the solvent and subsequent recrystallisation with ethanol resulted analytical samples of 3a-h. The scheme of the synthesis of title compounds is shown in Figure 1 and the Comparison of reaction times and yields of compound (3a-h) under microwave and classical methods is showing in Table 1.

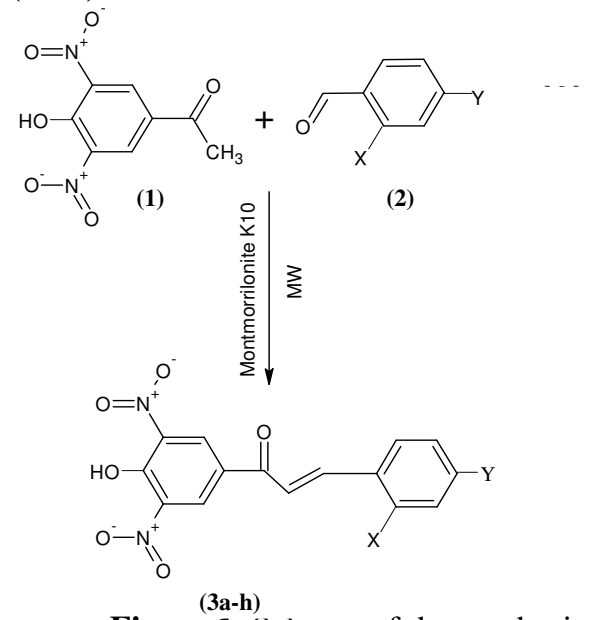

\begin{tabular}{ccl}
\hline Compounds & $\mathrm{X}$ & $\mathrm{Y}$ \\
\hline 3a & $\mathrm{H}$ & $\mathrm{OCH}_{3}$ \\
3b & $\mathrm{H}$ & $\mathrm{H}$ \\
3c & $\mathrm{Br}$ & $\mathrm{H}$ \\
3d & $\mathrm{H}$ & $\mathrm{Br}$ \\
3e & $\mathrm{Cl}$ & $\mathrm{H}$ \\
$\mathbf{3 f}$ & $\mathrm{H}$ & $\mathrm{Cl}$ \\
$\mathbf{3 g}$ & $\mathrm{H}$ & $\mathrm{OH}$ \\
3h & $\mathrm{H}$ & $\mathrm{CH}_{3}$ \\
\hline
\end{tabular}

Figure 1. Scheme of the synthesis of chalcones $(\mathbf{3 a}-\mathbf{h})$ 
Table 1. Comparison of reaction times and yields of compound (3a-h) under microwave and classical methods

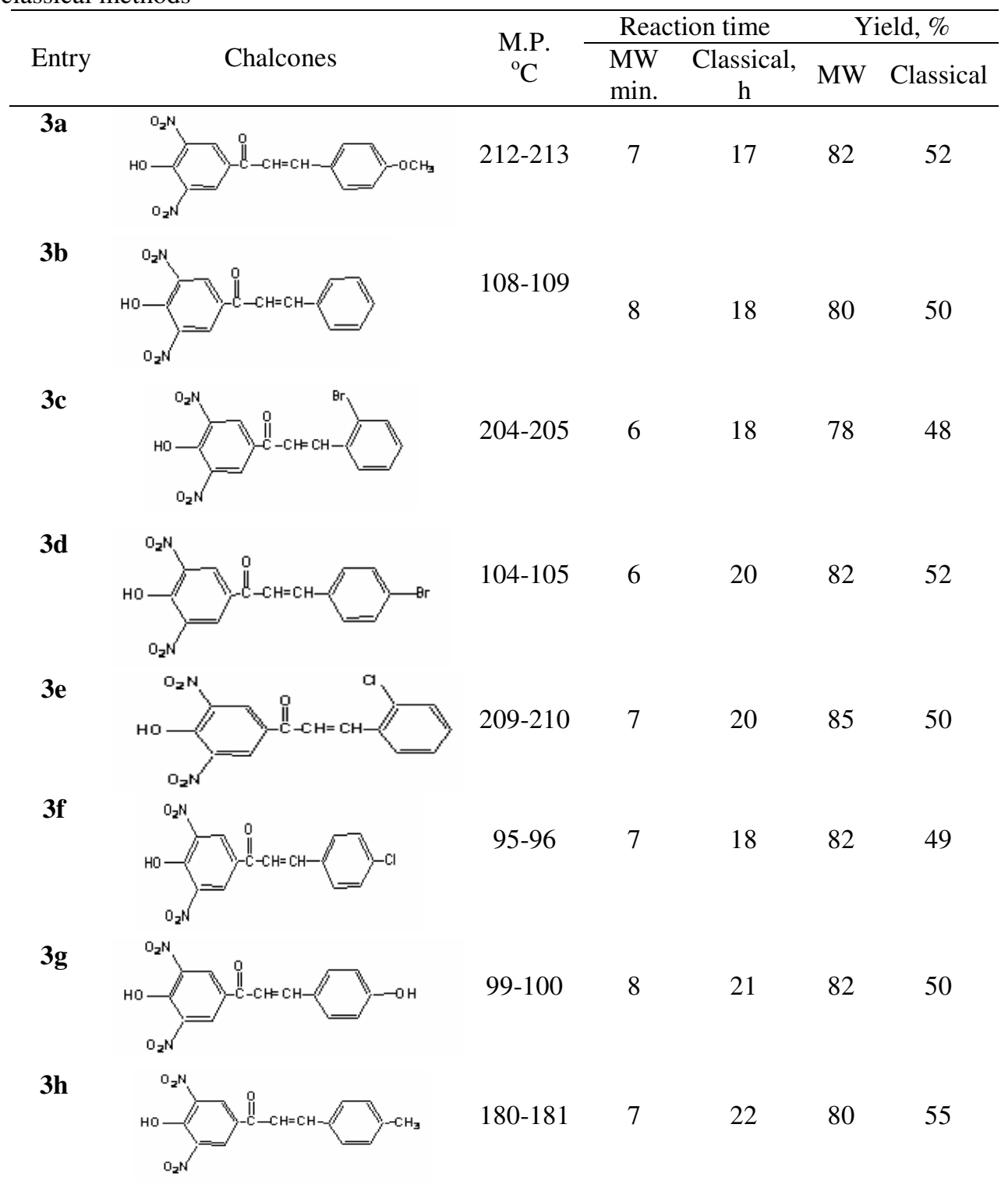

2(E)-1-(4-hydroxy-3,5-dinitrophenyl)-3-(4-methoxy phenyl)propenone (3a)

Dark yellow crystals, ${ }^{1} \mathrm{H}$ NMR $\left(500 \mathrm{MHz}, \mathrm{CDCl}_{3}\right) \delta: 8.9(\mathrm{~d} . \mathrm{J}=8,2 \mathrm{H}), 12.1(\mathrm{~s}, \mathrm{H}, \mathrm{OH}$ of acetophenone) $7.4(\mathrm{~d}, \mathrm{~J}=8,2 \mathrm{H}), 7.1(\mathrm{~d}, \mathrm{~J}=8,2 \mathrm{H}), 3.9\left(\mathrm{~s}, 3 \mathrm{H}, \mathrm{OCH}_{3}\right) 7.78(\mathrm{~d}, \mathrm{~J}=8, \mathrm{H})-\mathrm{COCH}=$, 7.51 (d, J=16, H, =CH-Ar); ${ }^{13} \mathrm{C}-\mathrm{NMR}\left(500 \mathrm{MHz}, \mathrm{CDCl}_{3}\right) \delta=26.46,77.01,114.71,116.29$, 130.77, 130.97, 147.65. IR (KBr) $/ \mathrm{cm}^{-1}: 3433 \mathrm{~cm}^{-1}(-\mathrm{OH}), 1664 \mathrm{~cm}^{1}(\mathrm{C}=\mathrm{O}), 1633 \mathrm{~cm}^{-1}$ $(\mathrm{CH}=\mathrm{CH}), 1118 \mathrm{~cm}^{-1}\left(-\mathrm{OCH}_{3}\right), 1358 \mathrm{~cm}^{-1}\left(-\mathrm{NO}_{2}\right)$ Anal.Calcd for $\mathrm{C}_{16} \mathrm{H}_{12} \mathrm{O}_{7} \mathrm{~N}_{2}: \mathrm{C}, 55.8 \%, \mathrm{H}$, $3.48 \%, \mathrm{~N}, 8.18 \%$.found $\mathrm{C}, 55.01 \%, \mathrm{H}, 3.25 \%, \mathrm{~N}, 8.13 \%$. 
(2E)-1-(4-hydroxy-3,5-dinitrophenyl)-3-(phenyl)propenone (3b)

Light red crystals, ${ }^{1} \mathrm{H}$ NMR $(500 \mathrm{MHz} \delta: 8.9$ (d.J=8,2H), 12.1(s,H, OH of acetophenone) 7.1$7.8(\mathrm{~m}, 5 \mathrm{H})$ Phenyl,7.75 (d,J=16,H) $-\mathrm{COCH}=, 7.5(\mathrm{~d}, \mathrm{~J}=16, \mathrm{H})=\mathrm{CH}-\mathrm{Ar} .{ }^{13} \mathrm{C} \mathrm{NMR}(500 \mathrm{MHz}$, $\left.\mathrm{CDCl}_{3}\right) \delta=26.27,77.05,128.94,129.20,130.69,137.51,152.51,192.57 . \mathrm{IR}(\mathrm{KBr}) / \mathrm{cm}^{-1}: 3432 \mathrm{~cm}^{-1}$ $(-\mathrm{OH}), 1693 \mathrm{~cm}-1 \quad(\mathrm{C}=\mathrm{O}), 1630 \mathrm{~cm}^{-1}(\mathrm{CH}=\mathrm{CH}), 1360 \mathrm{~cm}^{-1}\left(-\mathrm{NO}_{2}\right)$ Anal. Calcd for $\mathrm{C}_{15} \mathrm{H}_{10} \mathrm{O}_{6} \mathrm{~N}_{2}$ : C, 57.32\%, H, 3.18\%, N, 8.95\% found C, 55.18\%, H, 3.12\%, N,9.88\%.

(2E)-3-(2-bromophenyl)-3-(4-hydroxy-3,5-dinitrophenyl)propenone (3c)

Light yellow crystals, ${ }^{1} \mathrm{H}$ NMR $\left(500 \mathrm{MHz}^{\mathrm{C}} \mathrm{CDCl}_{3}\right) \delta: 8.9(\mathrm{~d} . \mathrm{J}=8,2 \mathrm{H}), 12.1(\mathrm{~s}, \mathrm{H}, \mathrm{OH}$ of acetophenone) 7.4(d, J=8,H),7.3(t, J=8, H), $7.2(\mathrm{t}, \mathrm{J}=8, \mathrm{H}), 7.5(\mathrm{~d}, \mathrm{~J}=8, \mathrm{H}), 7.85$ $(\mathrm{d}, \mathrm{J}=16, \mathrm{H})-\mathrm{COCH}=, 7.59(\mathrm{~d}, \mathrm{~J}=16, \mathrm{H})=\mathrm{CH}-\mathrm{Ar} .{ }^{13} \mathrm{C}$ NMR $\left(500 \mathrm{MHz}, \mathrm{CDCl}_{3}\right)$ $\delta=30.10,77.01,128.12,131.00,132.30,133.83,146.11,165.71,171.67 . \mathrm{IR}(\mathrm{KBr}) / \mathrm{cm}^{-1}$ : $3434 \mathrm{~cm}^{-1}(-\mathrm{OH}) 1668 \mathrm{~cm}^{-1}(\mathrm{C}=\mathrm{O}), 1632 \mathrm{~cm}^{-1}(\mathrm{CH}=\mathrm{CH}), 837 \mathrm{~cm}^{-1}(\mathrm{C}-\mathrm{Br}), 1352 \mathrm{~cm}^{-1}$ $\left(\mathrm{NO}_{2}\right)$ Anal. Calcd for $\mathrm{C}_{15} \mathrm{H}_{9} \mathrm{O}_{6} \mathrm{~N}_{2} \mathrm{Br}: \mathrm{C}, 45.8 \%, \mathrm{H}, 2.28 \%, \mathrm{~N}, 7.18 \%$.found $\mathrm{C}$, $45.48 \%, \mathrm{H}, 2.25 \%, \mathrm{~N}, 7.12 \%$.

\section{(2E)-3-(4-bromophenyl)-1-(4-hydroxy-3,5-dinitrophenyl) propenone (3d)}

Dark yellow crystals; ${ }^{1} \mathrm{H}$ NMR $\left(500 \mathrm{MHz}, \mathrm{CDCl}_{3} \delta: 8.9\right.$ (d. J=8, 2H), 12.1 (s, H, OH of acetophenone) $7.6(\mathrm{~d}, \mathrm{~J}=8,2 \mathrm{H}), 7.2(\mathrm{~d}, \mathrm{~J}=8,2 \mathrm{H}), 7.77(\mathrm{~d}, \mathrm{~J}=18, \mathrm{H})-\mathrm{COCH}=, 7.49$ $(\mathrm{d}, \mathrm{J}=16, \mathrm{H})=\mathrm{CH}-\mathrm{Ar} .{ }^{13} \mathrm{C}$ NMR $\left(500 \mathrm{MHz}, \mathrm{CDCl}_{3}\right) \delta=26.24,77.02,130.23,130.83,132.50$, 137.52, 152.22, 192.49. IR (KBr) $/ \mathrm{cm}^{-1}: 3430 \mathrm{~cm}^{-1}(-\mathrm{OH}), 1693 \mathrm{~cm}^{-1}(\mathrm{C}=\mathrm{O}), 1631 \mathrm{~cm}^{-1}$ $(\mathrm{CH}=\mathrm{CH}), 1360 \mathrm{~cm}^{-1}\left(-\mathrm{NO}_{2}\right), 848(\mathrm{C}-\mathrm{Br})$ Anal. Calcd for $\mathrm{C}_{15} \mathrm{H}_{9} \mathrm{O}_{6} \mathrm{~N}_{2} \mathrm{Br}: \mathrm{C}, 45.8 \%, \mathrm{H}$, $2.29 \%, \mathrm{~N}, 7.18 \%$. found $\mathrm{C}, 45.6 \%, \mathrm{H}, 2.25 \%, \mathrm{~N}, 7.12 \%$.

\section{(2E)-3-(2-chlorophenyl)-1-(4-hydroxy-3, 5-dinitrophenyl) propenone (3e)}

Light yellow crystals, ${ }^{1} \mathrm{H} \mathrm{NMR}\left(500 \mathrm{MHz}, \mathrm{CDCl}_{3}\right) \delta: 8.9(\mathrm{~d} . \mathrm{J}=8,2 \mathrm{H}), 12.1(\mathrm{~s}, \mathrm{H}, \mathrm{OH}$ of acetophenone) $7.5(\mathrm{~d}, \mathrm{~J}=8, \mathrm{H}), 7.55(\mathrm{t}, \mathrm{J}=8, \mathrm{H}), 7.40(\mathrm{t}, \mathrm{J}=8, \mathrm{H}), 7.77(\mathrm{~d}, \mathrm{~J}=8, \mathrm{H}), 7.85$ $(\mathrm{d}, \mathrm{J}=16, \mathrm{H})-\mathrm{COCH}=, 7.60(\mathrm{~d}, \mathrm{~J}=16, \mathrm{H})=\mathrm{CH}-\mathrm{Ar} .{ }^{13} \mathrm{C} \mathrm{NMR}\left(500 \mathrm{MHz}, \mathrm{CDCl}_{3}\right) \delta=$ $30.92,77.01,127.31,128.91,130.95,132.36,152.04,184.57, . \mathrm{IR}(\mathrm{KBr}) / \mathrm{cm}^{-1}: 3434 \mathrm{~cm}^{-1}$ $(-\mathrm{OH}), 1668 \mathrm{~cm}^{-1}(\mathrm{C}=\mathrm{O}), 1632 \mathrm{~cm}^{-1}(\mathrm{CH}=\mathrm{CH}), 825 \mathrm{~cm}^{-1}(\mathrm{C}-\mathrm{Cl}), 1363 \mathrm{~cm}^{-1}\left(-\mathrm{NO}_{2}\right)$ Anal. Calcd for $\mathrm{C}_{15} \mathrm{H}_{9} \mathrm{O}_{6} \mathrm{~N}_{2} \mathrm{Cl}: \mathrm{C}, 51.7 \%, \mathrm{H}, 2.58 \%, \mathrm{~N}, 8.05 \%$.found C, $51.48 \%, \mathrm{H}, 2.25 \%$, $\mathrm{N}, 8.03 \%$.

\section{(2E)-3-(4-chlorophenyl)-1-(4-hydroxy-3, 5-dinitrophenyl) propenone (3f)}

Dark yellow crystals, ${ }^{1} \mathrm{H}$ NMR $\left(500 \mathrm{MHz}, \mathrm{CDCl}_{3} \delta: 8.9\right.$ (d. J = 8,2H), $12.1(\mathrm{~s}, \mathrm{H}, \mathrm{OH}$ of acetophenone $) 7.6(\mathrm{~d}, \mathrm{~J}=8,2 \mathrm{H}), 7.4(\mathrm{~d}, \mathrm{~J}=8,2 \mathrm{H}), 7.78(\mathrm{~d}, \mathrm{~J}=16, \mathrm{H})-\mathrm{COCH}=, 7.50(\mathrm{~d}, \mathrm{~J}=$ $16, \mathrm{H})=\mathrm{CH}-\mathrm{Ar} .{ }^{13} \mathrm{C}$ NMR $\left(500 \mathrm{MHz}, \mathrm{CDCl}_{3}\right) \delta=26.27,77.04,128.08,128.89,130.68$, 137.51, 152.11, 192.56. IR (KBr) / cm $: 3432 \mathrm{~cm}^{-1}(-\mathrm{OH}), 1693 \mathrm{~cm}^{-1}(\mathrm{C}=\mathrm{O}), 1631 \mathrm{~cm}^{-1}$ $(\mathrm{CH}=\mathrm{CH}), 1360 \mathrm{~cm}^{-1}\left(-\mathrm{NO}_{2}\right), 814 \mathrm{~cm}^{-1}(\mathrm{C}-\mathrm{Cl})$ Anal.Calcd for $\mathrm{C}_{15} \mathrm{H}_{9} \mathrm{O}_{6} \mathrm{~N}_{2} \mathrm{Cl}: \mathrm{C}, 51.8 \%, \mathrm{H}$, $2.58 \%, \mathrm{~N}, 8.05 \%$.found $\mathrm{C}, 51.6 \%, \mathrm{H}, 2.23 \%, \mathrm{~N}, 8.03 \%$.

\section{(2E)-1-(4-hydroxy-3,5-dinitrophenyl)-3-(4-hydroxyphenyl) propenone $(\mathbf{3 g})$}

Dark yellow crystals, ${ }^{1} \mathrm{H}$ NMR $\left(500 \mathrm{MHz}, \mathrm{CDCl}_{3} \delta: 8.9(\mathrm{~d} . \mathrm{J}=8,2 \mathrm{H}), 12.1(\mathrm{~s}, \mathrm{H}, \mathrm{OH}\right.$ of acetophenone) $7.3(\mathrm{~d}, \mathrm{~J}=8,2 \mathrm{H}), 7.5(\mathrm{~d}, \mathrm{~J}=8,2 \mathrm{H}), 13.1(\mathrm{~s}, \mathrm{H}, \mathrm{OH}) 7.73(\mathrm{~d}, \mathrm{~J}=16, \mathrm{H}, \mathrm{COCH}=$, $7.49(\mathrm{~d}, \mathrm{~J}=16, \mathrm{H})=\mathrm{CH}-\mathrm{Ar} .{ }^{13} \mathrm{C} \mathrm{NMR}\left(500 \mathrm{MHz}, \mathrm{CDCl}_{3}\right) \delta=26.24,77.04,128.08,130.68$, 132.38, 137.52, 152.11, 192.59. IR (KBr) / cm $: 3420 \mathrm{~cm}^{-1}(-\mathrm{OH}), 1693 \mathrm{~cm}^{-1}(\mathrm{C}=\mathrm{O}), 1630 \mathrm{~cm}^{-1}$ $(\mathrm{CH}=\mathrm{CH}), 1360 \mathrm{~cm}^{-1}\left(-\mathrm{NO}_{2}\right), 3508 \mathrm{~cm}^{-1}(\mathrm{C}-\mathrm{OH})$ Anal. Calcd for $\mathrm{C}_{15} \mathrm{H}_{10} \mathrm{O}_{7} \mathrm{~N}_{2}: \mathrm{C}, 54.5 \%, \mathrm{H}$, $3.03 \%, \mathrm{~N}, 8.51 \%$.found $\mathrm{C}, 53.99 \%, \mathrm{H}, 2.98 \%, \mathrm{~N}, 8.48 \%$. 
(2E)-1-(4-hydroxy-3,5-dinitrophenyl)-3-(4-methyl phenyl)propenone(3h)

Spiny yellow crystals, ${ }^{1} \mathrm{H}$ NMR $\left(500 \mathrm{MHz}, \mathrm{CDCl}_{3}\right) \delta: 8.9$ (d.J=8,2H), 12.1 (s, H, OH of acetophenone) $7.2(\mathrm{~d}, \mathrm{~J}=8,2 \mathrm{H}), 7.56(\mathrm{~d}, \mathrm{~J}=8,2 \mathrm{H}), 2.35\left(\mathrm{~s}, 3 \mathrm{H},-\mathrm{CH}_{3}\right) 7.71(\mathrm{~d}, \mathrm{~J}=16, \mathrm{H})-\mathrm{COCH}=$, $7.50(\mathrm{~d}, \mathrm{~J}=16, \mathrm{H}=\mathrm{CH}-\mathrm{Ar}) .{ }^{13} \mathrm{C}$ NMR $\left(500 \mathrm{MHz}, \mathrm{CDCl}_{3}\right) \delta=30.93,77.02,129.97,130.68$, 130.84, 131.29, 148.54, 184.45. IR (KBr) / cm ${ }^{-1}: 3434 \mathrm{~cm}^{-1}(-\mathrm{OH}), 1665 \mathrm{~cm}-1(\mathrm{C}=\mathrm{O}), 1628 \mathrm{~cm}^{-1}$ $(\mathrm{CH}=\mathrm{CH}), 1366 \mathrm{~cm}^{-1}\left(-\mathrm{NO}_{2}\right), 2927 \mathrm{~cm}^{-1}\left(\mathrm{C}^{-\mathrm{CH}_{3}}\right)$ Anal. Calcd for $\mathrm{C}_{16} \mathrm{H}_{12} \mathrm{O}_{6} \mathrm{~N}_{2}: \mathrm{C}, 58.5 \%, \mathrm{H}$, $3.65 \%, \mathrm{~N}, 8.55 \%$ found $\mathrm{C}, 58.02 \%, \mathrm{H}, 2.98 \%, \mathrm{~N}, 8.52 \%$.

\section{Results and Discussion}

\section{Antimicrobial activity}

The newly synthesized compounds (3a-h) were screened for their antibacterial activity against Escherichia coli, Staphylococcus aureus and antifungal activity against Alternaria alternate by measuring the zone of inhibition in $\mathrm{mm}$. The antimicrobial activity was performed by cup plate method $^{28}$ at concentration $500 \mu \mathrm{g} / \mathrm{mL}$ and reported in Table 2. Nutrient agar and Czapex Dox agar was employed as culture medium for antibacterial and antifungal respectively. DMSO was used as solvent control for antimicrobial activity. Tetracycline and fluconazole were used as standard for antibacterial and antifungal activities respectively.

Table 2. Antimicrobial activity of synthesized compounds (3a-h)

\begin{tabular}{|c|c|c|c|}
\hline \multirow{3}{*}{ Compounds. } & \multicolumn{2}{|c|}{ Antibacterial } & Antifungal \\
\hline & \multicolumn{3}{|c|}{ Zone of inhibition in $\mathrm{mm}$} \\
\hline & E. coli & S. aureus & A. alternate \\
\hline $3 \mathbf{a}$ & 10 & 7 & 8 \\
\hline 3b & 6 & 4 & 6 \\
\hline 3c & 8 & 5 & 9 \\
\hline 3d & 12 & 7 & 13 \\
\hline $3 e$ & 11 & 8 & 5 \\
\hline $3 f$ & 13 & 10 & 6 \\
\hline $3 \mathbf{g}$ & 8 & 6 & 14 \\
\hline $3 h$ & 7 & 1 & 7 \\
\hline Terracycline & 16 & 13 & - \\
\hline Fluconazol & - & - & 16 \\
\hline
\end{tabular}

\section{Conclusion}

From the results of antimicrobial screening it was observed that all the compounds exhibited activity against all the microorganisms employed. According to the structure activity relationship, marked inhibition in bacteria was observed in the compounds (3a, 3c, 3d, 3f and $\mathbf{3 g}$ ) whereas other compounds show moderate to good activity. Antifungal screening data also revealed that compounds (3d, $\mathbf{3 g}$ ) imparted maximum activity to the compounds, whereas other compounds showed moderate to good activity.

\section{Acknowledgment}

The authors are thankful to Prof. B. L. Verma, Retd. Professor of Chemistry, M. L. S. University Udaipur, Dr. S. Baijal, Dean, FASC, MITS University and Mr. K. K. Maheshwari, G. M. Finance, MITS University for their constant encouragement during this work. Authors are also thankful to the Head, Sophisticated Analytical Instrument Facility, Indian Institute of Technology, Madras for spectral analysis. 


\section{References}

1. Vamakawa T, Kagechika H, Kawachi E, Hashimoto Y and Shedo K, J Med Chem., 1990, 33(5), 1430-1437.

2. Hogale M B, Dhore N P, Shelar A R and Pawar P K, Oriental J Chem., 1986, 2, 55-57.

3. Ahluwalia V K, Nayal L, Kaila N, Bala S and Tahim A K, Indian J Chem., 1987, 26B, 384-386.

4. Bhatt A K, Bhamaria R P, Patel M R, Bellare R A and Deliwala C V, Indian J Chem., 1972, 10, 694-698.

5. Mukharjee S, Kumar V, Prasad A K, Raj H G, Brakhe M E, Olsen C E, Jain S C and Parmar V P, Bioorg Med Chem., 2001, 9, 337-339.

6. Indyah S A, Timmerman H, Samhoedi M, Sastrohamidjojo S, Van Der Goot H, Eur J Med Chem., 2000, 35, 449-457.

7. Chen M, Cristensen S B, Zhai L, Rasmussen M H, Theander T G, Frokjaer S, Steffensen B, Davidson J and Kharazmi A, J Infect Dis., 1997, 176(5), 1327-1333.

8. Nielsen S F, Christensen S B, Cruciani G, Kharazmi A and Liefors T, J Med Chem., 1998, 41(24), 4819-4832.

9. Dhar D N, The Chemistry of Chalcones and Related Compounds, 1981 (John Wiley and Sons, New York)

10. Al-Arab M M, Ghanem B S and Fitton A O, Tetrahedron, 1989, 45, 6545-6452.

11. Patonay T, Levai A, Nemes C, Timar T, Toth G and Adam W, J Org Chem., 1996, 61, 5375

12. Rajendra Prasad Y, Lakshmana Rao A, Rambabu R and Ravi Kumar P, Oriental J Chem., 2007, 23(3), 927-937.

13. Srinivasa Rao M, Kotesh J, Marukulla R and Duddeck H, ARKIVOK, 2004, xiv, 96-102.

14. Akamatsu H, Fukasa K and Kusumoto S, Synlett., 2004, 6, 1049.

15. Varma R S, Tetrahedron. 2002, 58, 1235-1255

16. Ameta K L and Verma B L, J Indian Chem Soc., 2002, 79, 840.

17. Caddick S, Tetrahedron, 1995, 51, 10403-10432.

18. Calvino V, Picallo M, Lopez-Peinado A J, Martin-Aranda R M and Duran-Valle C J, Appl Surf Sci., 2006, 252(17), 6071-6074.

19. Kothari S, Vyas R and Verma B L, Indian J Heterocycl Chem., 1999, 8, 285.

20. Srivastava Y K, Sukhwal S, Ashawa A and Verma B L, J Indian Chem Soc., 1997, 71, 573.

21. Srivastava Y K and Verma B L, Nat Acad Sci Lett., 1987, 10, 319.

22. Kothari S, Singhal M, Vijayvergiya D, Vyas R and Verma B L, J Indian Chem Soc., 2000, 77, 329.

23. Srivastava Y K, Ameta K L and Verma B L, Indian J Heterocycl Chem., 2002, 11, 279.

24. Sharma P, Hussain K F, Sukhwal S, Kothari S, Singhal M and Verma B L, Indian J Chem., 1999, 38B, 966.

25. Rao S S, Gahlot U S, Dulawat S S, Vyas R, Ameta K L and Verma B L, Afinidad., 2003, 60(505), 271.

26. Gahlot U S, Rao S S, Dulawat S S, Ameta K L and Verma B L, Afinidad, 2003, 60(508), 558.

27. Gahlot U S, Rao S S, Jhala Y S, Dulawat S S and Verma B L, Indian J Heterocycl Chem., 2003, 13, 111.

28. Indian Pharmacopoeia, Ministry of Health and Family Welfare, New Delhi, 1996, A-114. 


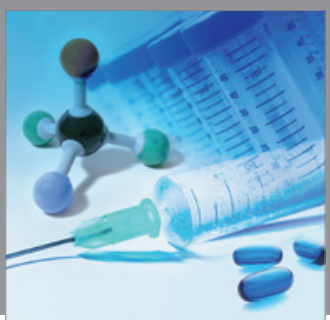

International Journal of

Medicinal Chemistry

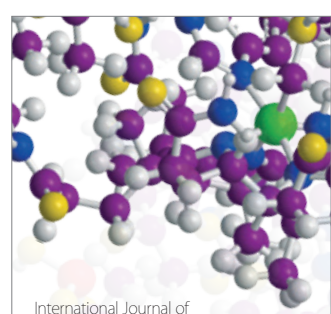

Carbohydrate Chemistry

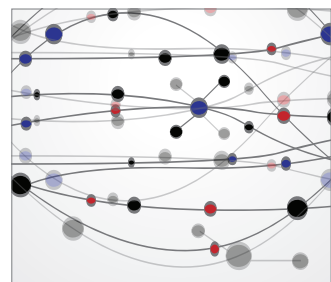

The Scientific World Journal
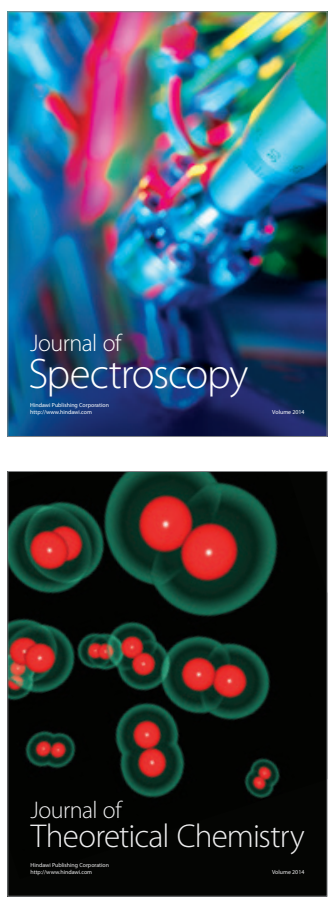
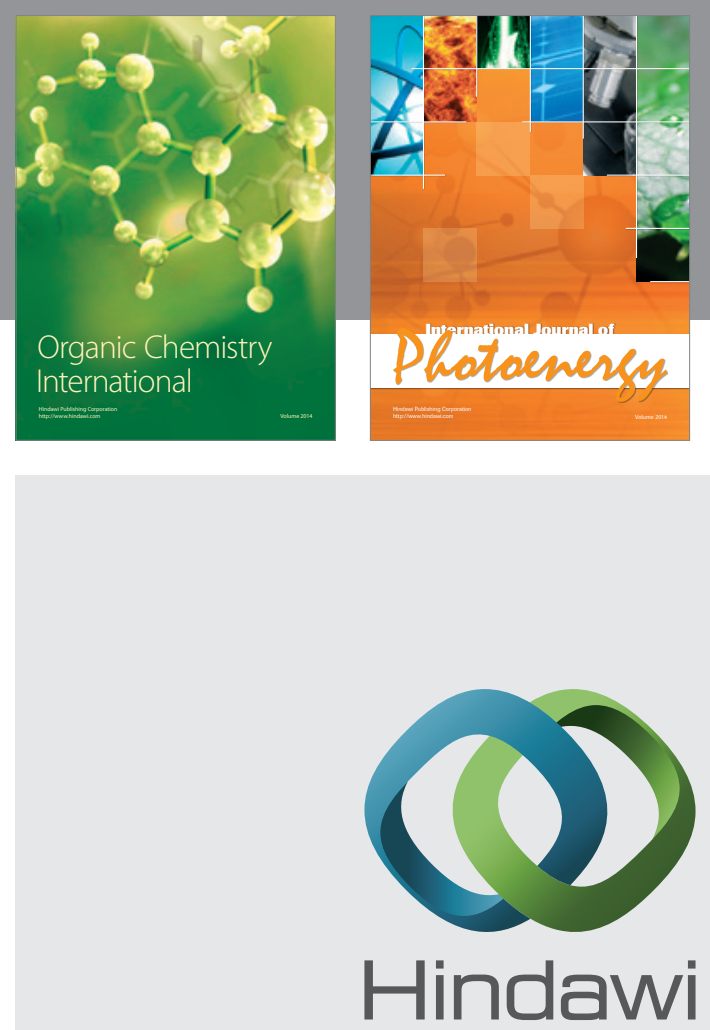

Submit your manuscripts at

http://www.hindawi.com
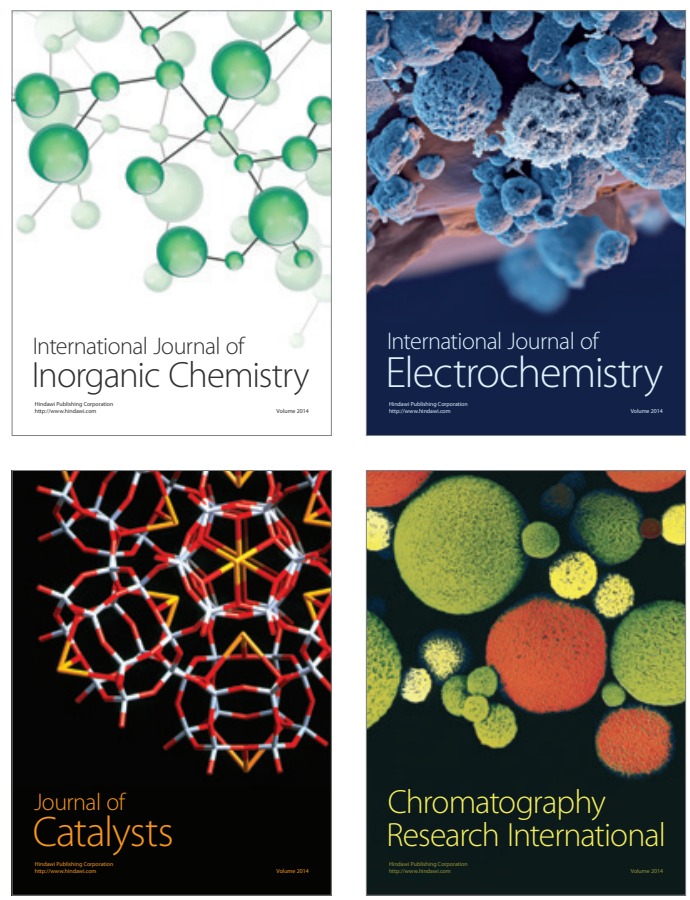
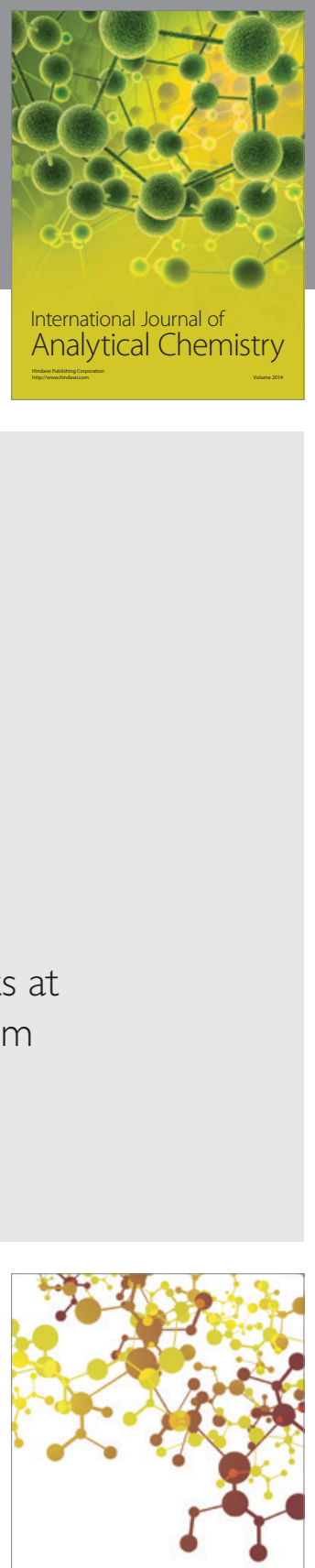

Journal of

Applied Chemistry
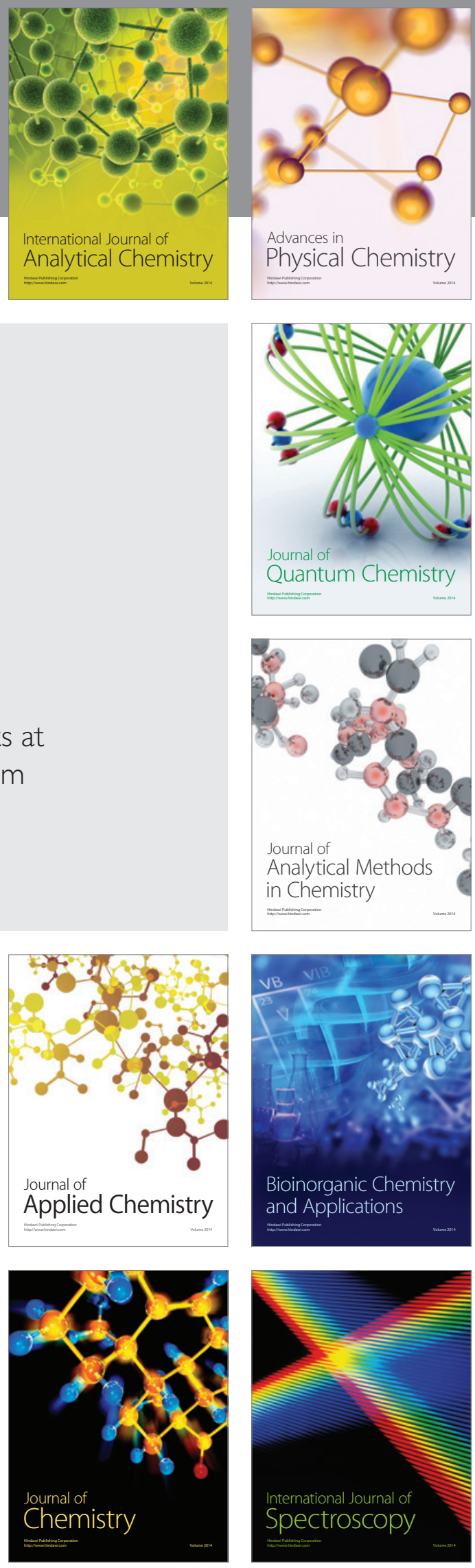